

\title{
Specific Approaches in Hormone-refractory Prostate Cancer Chemical treatment, surgery and patient evaluation
}

\author{
ADRIAN SILVIU CRISAN ${ }^{1 *}$, SORIN FLORESCU", MONICA SUSAN²*, AMADEUS DOBRESCU², IMOLA MIKLOS ${ }^{3}$, ALIN GRELUS ${ }^{3}$, \\ RAZVAN SUSAN ${ }^{2}$, DELIA BERCEANU VADUVA \\ 'Vasile Goldis Western University of Arad, 94 Revoluiei Blvd., 310025, Arad, Romania \\ ${ }^{2}$ Victor Babes University of Medicine and Pharmacy Timisoara, 2 Eftimie Murgu Sq., 300041, Timisoara, Romania \\ ${ }^{3}$ Arad County Emergency Hospital, 2-4 Andreny Karoly Str., 310037, Arad, Romania
}

In 2015 in Romania, a database for prostate cancer patients has been developed. The idea of the study started from the need to know the segment of patients diagnosed with hormone-refractory prostate cancer in Arad County. Patients were monitored from the time of diagnosis of prostate cancer by tracking clinical and biological evolution, with an emphasis on selecting those patients who developed the form of cancer that became resistant to castration, with the identification of urological complications and applied urological therapeutic methods in a palliative manner. In Romania, there is no screening program for prostate cancer, so most patients are diagnosed in the advanced local phase or in the metastatic phase. Because of this, treatment in prostate cancer is often reduced to chemical synthetic drug or urological palliative care.

Keywords: prostate cancer, evolution, histopathological evaluation

Prostate cancer ( $\mathrm{PCa}$ ) is one of the most common neoplasms in countries with increased life expectancy, the risk being directly proportional to age. The incidence of PCa in the European Union is 65 / 100,000 inhabitants / year and the mortality rate is $26 / 100,000$ inhabitants / year [1].

The Romanian Cancer League estimates that 177 new cases of prostate cancer are diagnosed annually and that about $7.3 \%$ of men die annually. Prostate cancer is the second cause of cancer death after lung cancer [2]. The average age at diagnosis is 71 years. In patients deceased by other causes, localized PCa was detected in autopsy in $8 \%$ of males aged $20-29$ and $83 \%$ of those in the $70-79$ age group [3]. The screening of healthy men using specific prostate antigen (PSA) leads to PCa detection. Chemoprevention with finasteride (Type II 5- $\alpha$-reductase inhibitor) can prevent low or medium grade $\mathrm{PCa}$, but increases the incidence of high grade PCa [4].

An immense and ongoing challenge is the early detection of prostate cancer and the application of the right treatment as well as the finding of palliative effective treatment, ideal of locally advanced hormone-refractory prostate cancer. In most cases, PCa has a slow evolution with discrete symptomatology, being curable at the localized stage, but in some cases it can aggressively evolve, becoming hormone-refractory (resistant to castration) [5] .

Patient quality of life is severely impaired by complications (chronic renal failure, unilateral or bilateral ureterohydronephrosis, urinary retention, etc.) caused by both local and distal spread of prostate cancer. They lead to the appearance of paraneoplastic symptoms, such as unilateral or bilateral lumbar pain through obstructive uropathy, generalized asthenia, cachexia, decreased muscle strength, digestive symptoms (nausea, vomiting, in appetence).

In patients with hormone-refractory prostate cancer, the most common manifestation is obstructive uropathy, the long-term effects of which depend on the severity of the obstruction, the onset of the onset, the single- or bilateral development of the neoplasm and the patient's basic kidney function. Bilateral obstruction is reflected in a series of changes in renal function. Initially, renal blood flow increases in both kidneys, but within 90 minutes there is a dramatic decrease in renal perfusion and glomerular filtration rate. These phenomena occur due to the accumulation of vasoactive substances: angiotensin II, thromboxane $\mathrm{A} 2$ and atrial natriuretic factor. The alteration of the tubular function also occurs. Ability to concentrate urine diminishes due to the loss of the medullar interstitial hypertonic gradient and the aquaporin water channel disruption. If obstruction is not corrected, release of cytokines and growth factors ( $\beta$ tumor growth factor, angiotensin II, NFKB and tumor necrosis factor $\alpha$ ) occurs, causing inflammation and subsequent fibrosis. It has been shown that recovery of renal function is inversely proportional to the period of obstruction. Upon raising the obstacle, glomerular filtration rate recovery after two weeks of obstruction was 70\%, after four weeks 30\%, and after six weeks renal function did not improve [6].

Acute obstruction of the upper urinary tract is manifested by renal colic, nausea, vomiting. Anuria may also occur in the case of obstruction of both ureters, or in case of single kidney (functional, surgical, and congenital) [6] .

Most often, extrinsic compression is a long process that gradually leads to chronic renal failure. Clinical symptoms are vague and include lumbar pain, anorexia, lethargy, mood swings. Due to the lack of specific symptomatology in locally advanced prostate cancer, urinary tract damage is insidious and ureterohydronephrosis is accidentally detected.

Urological therapeutic methods are widely used in the palliative treatment of patients with hormone refractory prostate cancer, and are needed to improve the overall condition of patients and prolong survival.

\section{Experimental part}

Materials and methods

The prospective study is conducted over a period of 4 years from 2013 to 2016 and includes a batch of 71 patients who have been hospitalized and treated in the Urology 
Clinic of the Arad County Emergency Clinical Hospital. Patients enrolled in the study were diagnosed with CRPC and followed dynamically in terms of evolution following the application of palliative urological methods. It has been followed and to what extent these methods increase life expectancy and increase the quality of the survival.

Criteria for inclusion in the study. a) Patients who have signed informed consent for participation in the study; b) Patients diagnosed with CRPC - as defined by CRPC in the European Guidelines for Urology; c) The presence of two or more new bone lesions detected by radio-imaging methods and d) Patients who developed urological complications.

Histopathological examination. Tissue samples were collected from each patient and were analyzed by conventional optical microscopy or immunohistochemical methods.

The Ethics Commission of the Arad County Emergency Clinical Hospital and the Scientific Research Ethics Committee of the Vasile Goldis West University approved this study, which has complied with all ethical and ethical norms, as well as the Helsinki Declaration for studies with human subjects.

\section{Results and discussions}

Patients were monitored from the time of diagnosis of prostate cancer by tracking clinical and biological evolution, with an emphasis on selecting those patients who developed the form of cancer that became resistant to castration, with the identification of urological complications and applied urological therapeutic methods in a palliative manner. Prostate cancer becomes hormonerefractory after a 3-5 year evolution. First-line drug therapy for hormone-refractory prostate cancer is the four FDAapproved medicines for use in patients with CRPC, namely: docetaxel in combination with prednisone, cabazitaxel, abiraterone acetate and sipuleucel-T. Palliative surgery is required to relieve symptoms and ensure the quality of life of the patient and becomes a reasonable alternative when healing is not possible or when attempts to achieve healing result in adverse reactions that the patient cannot tolerate. In all these situations, urological therapeutic methods are needed to alleviate complications of advanced prostate cancer.

Histopathologically, actual, the following forms of prostate cancer are distinguished: Small cell carcinoma; Mucinous adenocarcinoma; Adenocarcinoma with signet ring cell-like features (cell in seal ring); Ductal adenocarcinoma; Basal cell carcinoma; Acinar adenocarcinoma, which presents the following histopathological changes - mucinous fibroplasia, carcinoma foamy gland, Neuroendocrine differentiation Paneth-like cells; Prostate adenocarcinoma treated by radiotherapy; Pseudohyperplastic prostatic adenocarcinoma; Intraductal prostatic carcinoma [7].

Histopathological results help to determine the prognosis and grouping of patients by the Gleason score and disease status group provided by the TNM classification.

The Gleason score is based exclusively on the microscopic aspects of tumor glands at small targets. Initially, only Gleason grades were defined, which were defined from 1 to 5 (grade 1 being the most differentiated model, and grade 5 poorly differentiated or undifferentiated carcinoma), then the Gleason score was introduced, which aims at identifying two architectural models - the primary model, predominantly the model of secondary importance [8].
Gleason grades are:Grade 1 - cancerous tissue is very similar to normal prostatic tissue; grade 2 - the presence of well-defined glands, but of larger size; grade 3 - darker cells, go beyond the glandular structure and invade neighboring tissue; grade 4 - very few glands, invasion of neighboring tissue; grade 5 - absent glands.

Interpretation of the Gleason score: Gleason 2-4 - well differentiated tumor; Gleason 5-7 - moderately differentiated tumor; Gleason 8-10- undifferentiated tumor [9].

It is considered that the prognosis of the disease is influenced by both primary and secondary architectural aspects, they are summed up resulting in a combined Gleason grade - Gleason score [9], so the Gleason score is higher, the more serious the neoplastic disease is.

Looking at the study as a whole, there are 6 patients, 2 with Gleason score 5 and 4 with Gleason score of 6 who were included in risk group 1, indicating a less aggressive form of neoplasia (see histopathological examination in figs. 1, 2). Current discussions about prostate cancer with Gleason score d" 6 are related to the need to initiate antiandrogenic therapy or to apply active surveillance to the patient. Some scientists consider that these grades should notbe treated. However, we should note that in our study there are patients with Gleason score $\leq 6$ and $8.45 \%$ of the total of 71 cases that have turned into hormonerefractory prostate cancer. This may be due either to the possibility of negative progression of prostate cancers with Gleason score $\leq 6$, or omission during prostate biopsy or

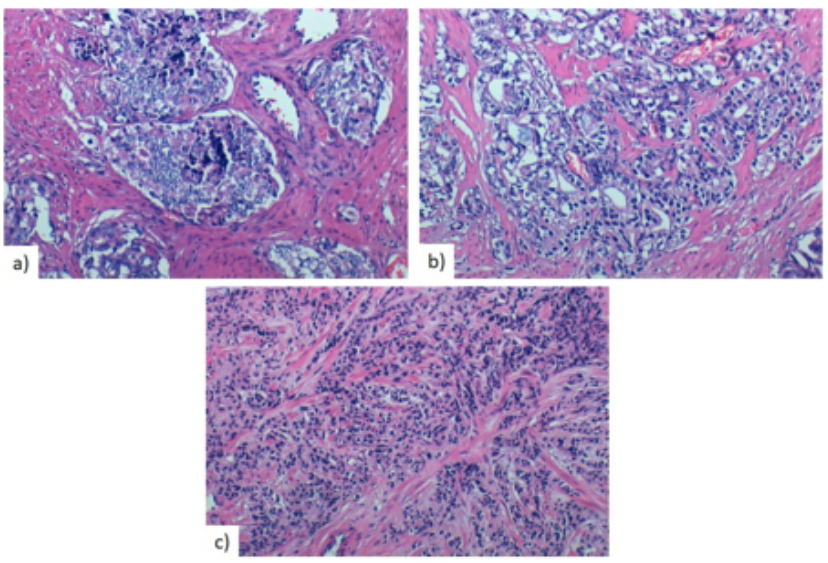

Fig.1a) A poorly differentiated prostate adenocarcinoma in a TURP specimen with solid cell lines and rare lumens. HEx100;

b)- Prostate adenocarcinoma with fused glands (pattern 4 Gleason). HEx200; c) - Biopsy puncture specimen with prostatic

adenocarcinoma with growth in trabeculae and individual tumor cells (pattern 5 Gleason). HEx 200

surgical intervention of a prostate cancer with a higher Gleason score.

Even if we have a total of 37 patients with a Gleason score of 7, their prognosis is different depending on the primary and secondary architectural aspects of the tumor. Thus, we have 11 patients with a Gleason score of 7 ( $3+$ 4) falling within prognostic group 3, with a $15.49 \%$ score. Unfortunately, in this study, the majority of patients, 26 patients - $36.61 \%$, were included in prognostic group 4 of patients with Gleason score of $7(4+3)$. Within the prognostic group 5 a number of 28 patients were enrolled, representing $39.44 \%$ and a number of 22 patients with Gleason score 8 and 6 patients with Gleason 9 score, almost maximal were identified. Looking at the whole group of patients with CRPC, we have a total of $76.04 \%$ including the patients with a Gleason score of $7(4+3), 8$, 9 , which in terms of prognosis is in the one with aggressive evolution. This percentage is considered high and should 

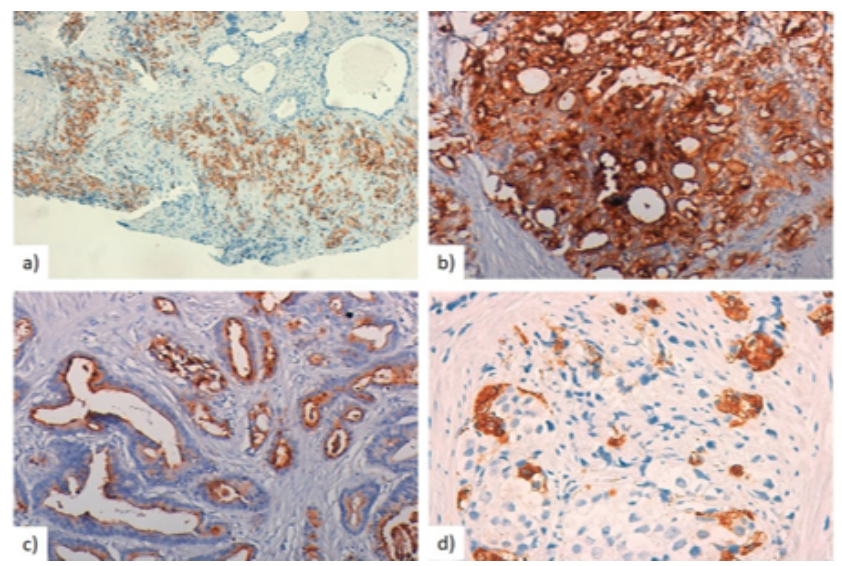

be taken into account particularly in terms of palliative methods thathad to be recommended along the course of the disease.

\section{Conclusions}

The main conclusions of this study are the following: the provenance environment is not a risk factor for the development of CRPC; patients with CRPC have a number of complications, among which the most serious is uniand / or bilateral ureterohydronephrosis, which evolves to IRC; most patients diagnosed with CRPC were classified as unfavorable prognostic groups; patients in the adverse prognostic groups had more frequent secondary anemia and bone metastases; anemia that occurs in patients with CRPC causes an increase in the prevalence of comorbid associations; the most recommended oncological treatment was total androgenic blockade.
Fig. 2a) Positive reaction for PSA in infiltrating tumor cells from a poorly differentiated adenocarcinoma with pattern 5 Gleason in the image (score 9 tumor). Anti-PSA, Ultravision, DAB, hematoxylin counter-x100; b) - Intensive positive reaction for PSMA in infiltrating tumor cells in a poorly differentiated adenocarcinoma (score 9). Anti-PSMA, Ultravision, DAB, hematoxylin counterclockwise, x200; c) Positive reaction for PSMA, predominantly apical, in an adenocarcinoma area with individualized, focal cribriform glands. Anti-PSMA, Ultravision, DAB, hematoxylin counterclockwise, x200; d) Disrupted and clustered neuroendocrine cells in a solid growth prostate adenocarcinoma. Anti-CgA, Ultravision, $\mathrm{DAB}$, hematoxylin counterclockwise, $x 400$

\section{References}

1. EISBRUCH, A., SHIP, J.A., DAWSON, L.A. et al. World J Surg. 27, 2003, p. 832

2. BERISHA, R., GEORGESCU, A., BONDARI, A., BOUTSIADIS, N., BOUTSIADIS, K. Current Health Sciences J ournal 36(4), 2010, p. 206. 3. *** https://www.rtog.org/ClinicalTrials/ProtocolTable/

StudyDetails. aspx?study=00224.

4. ANTES, K., DARBY, M., SONG, S., STARKSCHALL, G. Med Dosim. 24, 1999, p. 255.

5. *** http://uroweb.org/wp-content/uploads/EAU-GuidelinesProstate-Cancer-2015-v2.pdf

6. FRIEDLANDER, J.I., DUTY, B.D., OKEKE, Z., SMITH, A.D. Journal of Endourology, 26(2), 2012

7. KRYVENKO, O.N., EPSTEIN, J.I. Arch Pathol Lab Med. 140, 2016

8. EPSTEIN, J.I., ZELEFSKY, M.J., SJ OBERG, D.D. et al. European Urology 69, 2016, p. 428.

9. DEMARZO, A.M., NELSON, W.G., ISAACS, W.B., EPSTEIN, J.L. Lancet 15,2003, p. 955.

Manuscript received: 15.02 .2017 
